nephron

Practice
Nephron 2020;144:67-71

DOI: $10.1159 / 000504852$
Received: September 5, 2019 Accepted: November 17, 2019 Published online: December 11, 2019

\title{
The 21st UK Renal Registry Annual Report: A Summary of Analyses of Paediatric Data in 2017
}

\author{
Lucy Plumb ${ }^{a, b}$ Anna Casula ${ }^{a}$ Rhodri Pyart ${ }^{a}$ Katharine M. Evans ${ }^{a}$ \\ Carol Inward ${ }^{c}$ James Medcalfa Stephen D. Marks ${ }^{d, e}$
}

a UK Renal Registry, Bristol, UK; b Population Health Sciences, University of Bristol Medical School, Bristol, UK; ${ }^{c}$ Department of Paediatric Nephrology, University Hospitals Bristol NHS Foundation Trust, Bristol, UK; ${ }^{d}$ Department of Paediatric Nephrology, Great Ormond Street Hospital for Children NHS Foundation Trust, London, UK; e University College London Great Ormond Street Institute of Child Health, NIHR Great Ormond Street Hospital Biomedical Research Centre, London, UK

\section{Introduction}

Children with end-stage kidney disease (ESKD) requiring renal replacement therapy (RRT) are usually managed in one of the 13 paediatric nephrology centres in the United Kingdom. The UK Renal Registry (UKRR) collects, analyses and reports data on children receiving RRT in these centres.

The UKRR annual report presents analyses relating to the attainment of the Renal Association audit measures [1], national averages to enable benchmarking and longterm trends for children on RRT for ESKD. Data are reported by centre to enable between centre comparisons.

To improve the timeliness of data reporting, the format of the UKRR 21st Annual Report, which included data to December 31, 2017 [2], differs significantly to that of previous years. For paediatric data, changes include a single chapter reporting demographic and biochemical data, reduced clinical commentary, a higher threshold for data quality control (with data completeness at least 70\%) and greater alignment of analyses to the Renal Association guidelines [1].

\section{KARGER}

(c) 2019 S. Karger AG, Basel

E-Mail karger@karger.com

www.karger.com/nef
In this article, we summarise the analyses of paediatric data presented in the UKRR 21st Annual Report. The corresponding adult summary is published separately in this issue. The full UKRR 21st Annual Report can be accessed at https://www.renalreg.org/reports/data_to_end_2017/.

\section{Materials and Methods}

The paediatric data chapter of the UKRR 21st Annual report described children aged $<18$ years of age with ESKD who were on RRT in the United Kingdom for at least 90 days in 2017 under the care of paediatric renal centres. Children with ESKD are managed within a paediatric centre until transition to an adult centre, which occurs in general between 16 and 18 years of age. Young people aged 16-18 years may therefore be managed in either paediatric or adult services. This varies across the United Kingdom and is dependent on many factors, including local practices, social factors and child/family wishes. Consequently, demographic data have focused on children aged $<16$ years because this represents a more complete cohort, whereas attainment of the Renal Association guidelines is reported for children aged $<18$ years managed in paediatric centres.

All 13 paediatric nephrology centres in the United Kingdom contribute data to the UKRR. As in previous years, data items were 


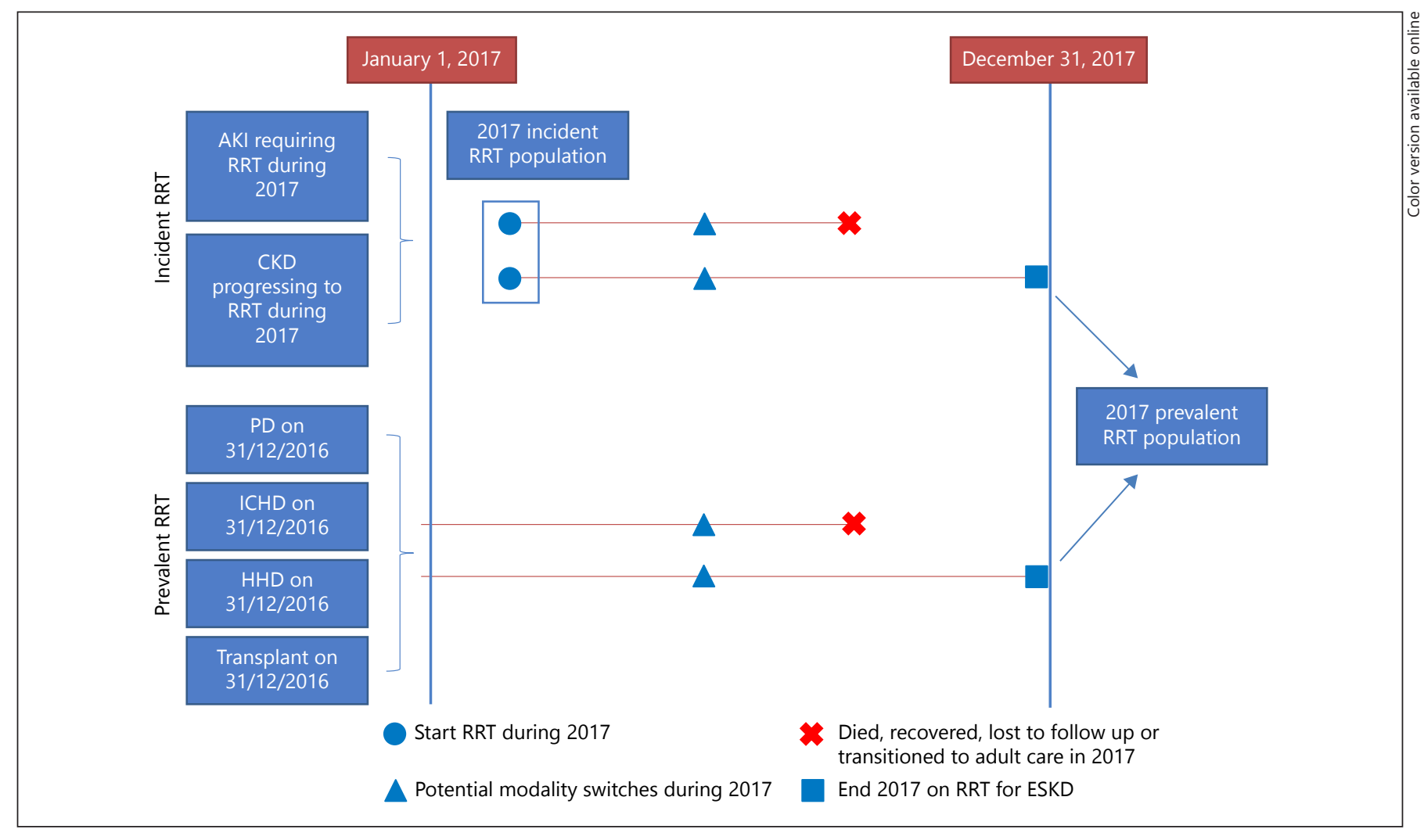

Fig. 1. An example of a patient cohort diagram used in the 21st UKRR Annual Report - pathways paediatric patients could follow to be included in the UK 2017 incident and/or prevalent RRT populations. AKI, acute kidney injury; RRT, renal replacement thera- py; CKD, chronic kidney disease; $\mathrm{PD}$, peritoneal dialysis; ICHD, in centre haemodialysis; HHD, home haemodialysis; ESKD, endstage kidney disease.

$\mathrm{mmol} / \mathrm{L}$ if aged 9 years or more was used. More details about the methods underlying the UKRR 21st Annual Report can be accessed in appendix A at https://www.renalreg.org/wp-content/ uploads/2019/05/21st_UKRR_Annual_Report_AppA.pdf

\section{Results}

In 2017,99 children $(67.7 \%$ male $)$ aged $<16$ years and with a median age of 7.6 years (interquartile range [IQR] 2.6-12.6 years) started RRT for ESKD, compared with 112 children the previous year. This gave an incidence of 7.9 per million age-related population (pmarp). No trend has been noted in the number of children starting RRT for ESKD in the United Kingdom, with annual incidence rates ranging from 7.9 to 10.2 pmarp over the past 5 years. For incident children in 2017, 42.4\% started RRT on peritoneal dialysis (PD), 35.4\% on haemodialysis (HD) and $22.2 \%$ received a pre-emptive kidney transplant. A fifth of incident children (20.2\%) in 2017 were late pre-
68

Nephron 2020;144:67-71

DOI: $10.1159 / 000504852$
Plumb/Casula/Pyart/Evans/Inward/ Medcalf/Marks 
Fig. 2. Start RRT modality for paediatric patients ( $<16$ years old) incident to RRT by 5 year time period. PD, peritoneal dialysis; $\mathrm{HD}$, haemodialysis; RRT, renal replacement therapy.

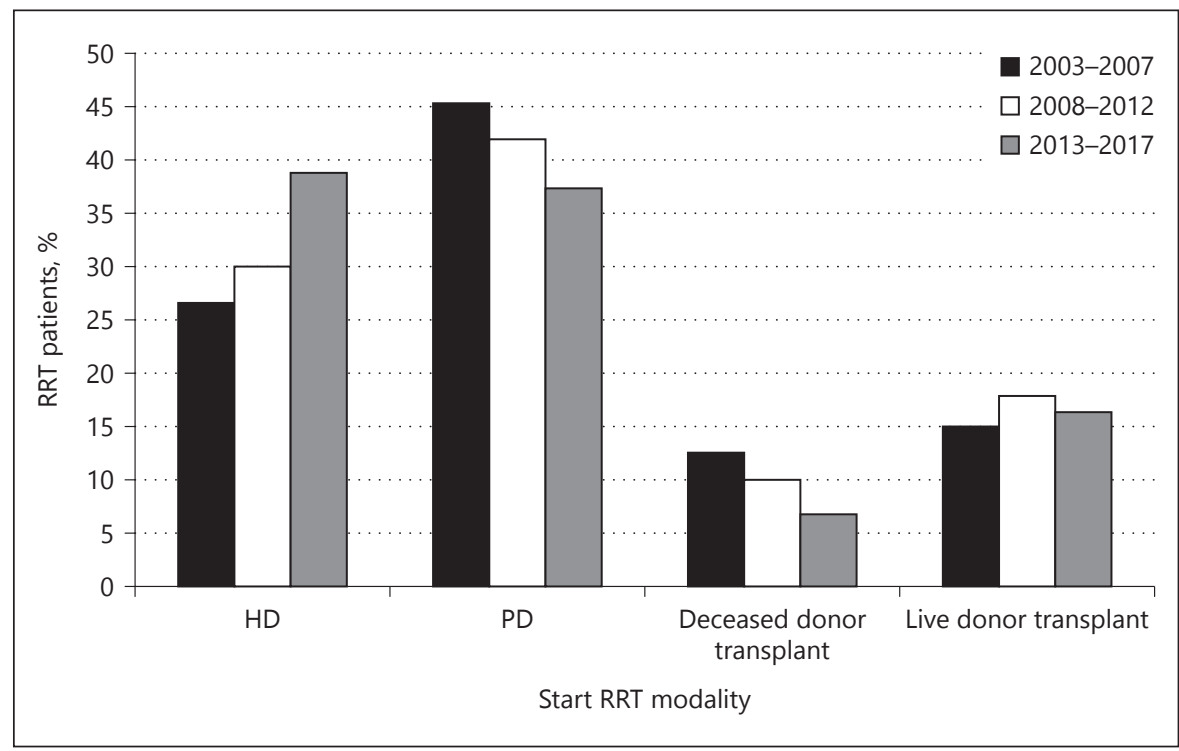

senters, starting RRT within 90 days of first nephrology review.

When incident data were examined by time period, greater proportions of non-White children (31.5\%) and those aged $<4$ years $(23.3 \%)$ were noted in the most recent 5-year period (2013-2017) than previously. Congenital anomalies of the kidneys and urinary tract accounted for almost half of all incident cases of RRT in children: this proportion has been stable over time. In 2013-2017, an increase in the proportion of children with miscellaneous kidney disorders was noted, contributing $13.3 \%$ of incident cases. Over the past 15 years, there have been increases in the proportion of children using HD as their first RRT modality: 2013-2017 was the first time period where HD use at RRT start surpassed that of PD. Live donor pre-emptive kidney transplantation has remained stable, while the proportion of children who received a deceased pre-emptive donor kidney transplant has fallen (Fig. 2).

Excluding children aged $<3$ months and late-presenting children (presenting to specialist kidney services within 90 days of RRT start), $33.8 \%(n=420)$ of incident children aged $<16$ years between 2003 and 2017 received a pre-emptive kidney transplant. Few Black (11.1\%) or South Asian (23.1\%) children received pre-emptive transplants, as did children aged $<2$ years $(4.4 \%)$ or those who were diagnosed with a glomerular disease (7.3\%).

As of December 31,2017, 810 children aged $<16$ years (966 under 18 years) were receiving RRT for ESKD in one of the 13 dedicated paediatric nephrology centres, a prevalence of 64.8 pmarp. Of this group, 522 (64.4\%) were male and 555 (69.0\%) were White. Most children (76.3\%) had a functioning transplant (45.1\% live, $31.2 \%$ deceased donor), with $13.1 \%$ on HD and $10.6 \%$ on PD. Of the 96 young people who transitioned to adult services, $84.4 \%$ had a functioning kidney transplant. Over time, increasing prevalence in children is noted: from 52.8 pmarp in 2013 to 64.8 pmarp in 2017.

Using z-scores standardised to the general UK childhood population, poorer growth, both in terms of height and weight attainment, was seen for children requiring RRT. This was more pronounced for prevalent children on dialysis compared to those with a functioning transplant. Prevalent children on dialysis had a median height z-score of -1.9 (IQR -2.95 to -0.9 ) compared with -1.1 for those with a functioning transplant (IQR -2.09 to $-0.3)$. The median weight $\mathrm{z}$-score for children on dialysis was -1.1 (IQR -2.13 to -0.4 ) compared with -0.1 (IQR -1.16 to 0.8$)$ for those with a functioning transplant. All centres had IQRs for height and weight that spanned the UK median.

The prevalence of CVRFs collated by the UKRR was reported: this included BMI, total cholesterol and systolic and diastolic blood pressure measurements. Analysis of these data was restricted to the 553 of 966 (57.2\%) children aged $<18$ years with data for all 3 risk factors, a similar proportion compared with the previous year (57.4\%). Of those with complete data, over a quarter of children (28.6\%) had no recorded CVRF, 37.2\% had 1 CVRF, and $34.2 \%$ had 2 or more CVRFs. In 2017, the prevalence of hypercholesterolaemia was similar to high BMI, affecting 37.8 and $37.5 \%$ of children, respectively. 
Fig. 3. Unadjusted Kaplan-Meier survival (from day 90) of incident paediatric RRT patients ( $<16$ years old) between 2003 and 2016 by age group at start of RRT.

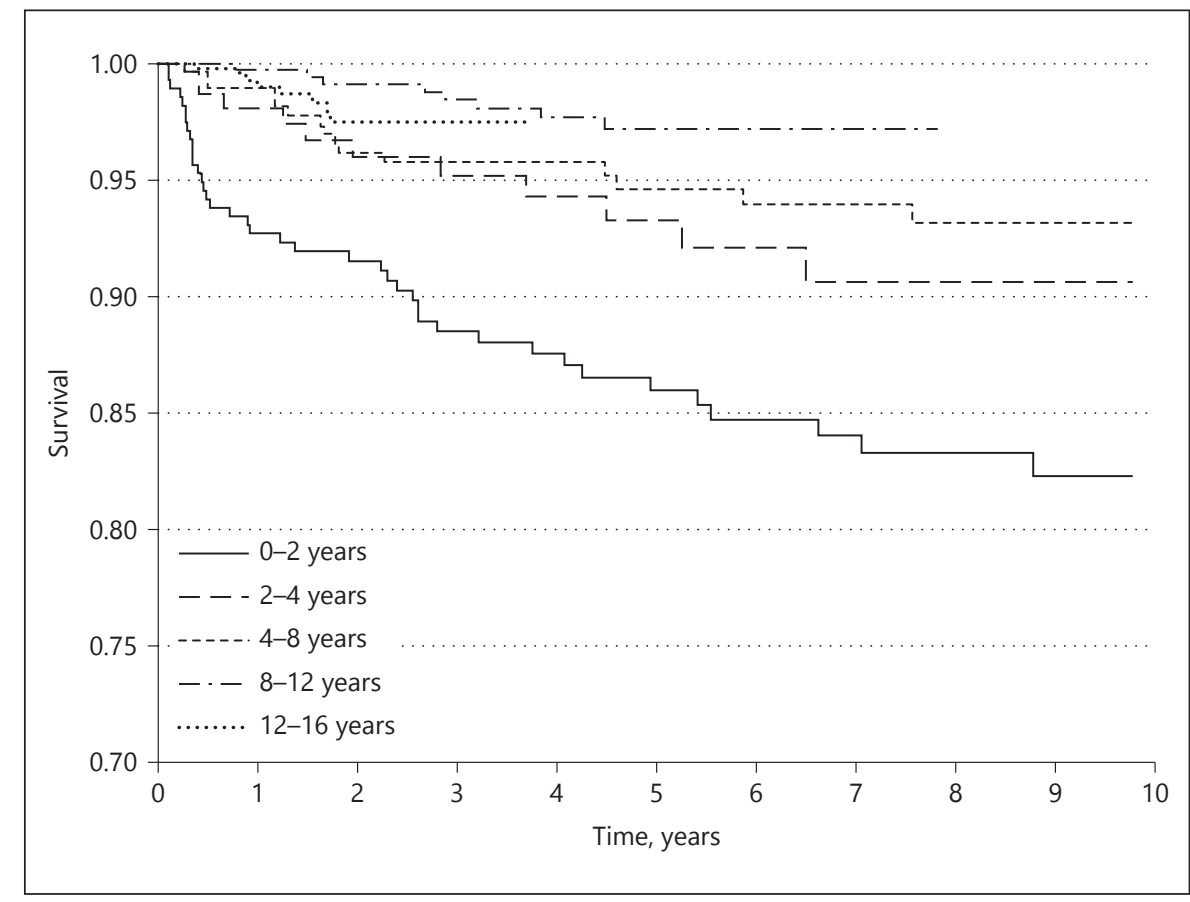

As of December 31, 2017, the median systolic blood pressure z-score reported for prevalent children on dialysis was 1.0 (IQR 0.19-2.1) and 0.4 (IQR -0.24 to 1.0) for transplanted children. A systolic blood pressure target of less than the 90th percentile was achieved by 53.3, 60.0 and $82.9 \%$ of HD, PD and transplanted children, respectively. For diastolic blood pressure, the same target was achieved in 55.6, 66.7 and 76.5\% of HD, PD and transplanted children, respectively.

This year's report focused on reporting estimated glomerular filtration rate (eGFR) using the Schwartz calculation [3] for transplant recipients, as opposed to creatinine values. The median eGFR for children with a functioning transplant $(n=749)$ was $60 \mathrm{~mL} / \mathrm{min} / 1.73 \mathrm{~m}^{2}$ (IQR $48-76 \mathrm{~mL} / \mathrm{min} / 1.73 \mathrm{~m}^{2}$ ); the proportion of transplanted children with an eGFR of $<30 \mathrm{~mL} / \mathrm{min} / 1.73 \mathrm{~m}^{2}$ varied across centres and ranged from 0 to $13.9 \%$. Median eGFR was also reported by age and time since transplant for children with available data. Younger children tended to have higher median eGFRs at any given time point: overall median eGFRs were $85 \mathrm{~mL} / \mathrm{min} / 1.73 \mathrm{~m}^{2}$ (IQR $62-105 \mathrm{~mL} / \mathrm{min} / 1.73 \mathrm{~m}^{2}$ ) in children aged $<5$ years compared with $52 \mathrm{~mL} / \mathrm{min} / 1.73 \mathrm{~m}^{2}$ (IQR $39-66 \mathrm{~mL} /$ $\mathrm{min} / 1.73 \mathrm{~m}^{2}$ ) in $16-18$ year olds. At 1 -year post-transplant, the median eGFR was $84 \mathrm{~mL} / \mathrm{min} / 1.73 \mathrm{~m}^{2}$ (IQR $54-97 \mathrm{~mL} / \mathrm{min} / 1.73 \mathrm{~m}^{2}$ ) for children aged $<5$ years, 69 $\mathrm{mL} / \mathrm{min} / 1.73 \mathrm{~m}^{2}$ (IQR $56-82 \mathrm{~mL} / \mathrm{min} / 1.73 \mathrm{~m}^{2}$ ) for 5 to
$<12$ year olds, $63 \mathrm{~mL} / \mathrm{min} / 1.73 \mathrm{~m}^{2}$ (IQR $52-73 \mathrm{~mL} /$ $\mathrm{min} / 1.73 \mathrm{~m}^{2}$ ) for 12 to $<16$ year olds and $53 \mathrm{~mL} / \mathrm{min} / 1.73$ $\mathrm{m}^{2}$ (IQR $52-66 \mathrm{~mL} / \mathrm{min} / 1.73 \mathrm{~m}^{2}$ ) for 16 to $<18$ year olds. Declines in eGFR were noted with time across all age groups: between 1 and 5 years post-transplant, smaller percentage losses in eGFR were noted in older compared with younger children.

Attainment of biochemical parameters was reported for prevalent children on dialysis $(n=217)$ and transplanted children with an eGFR of $<30 \mathrm{~mL} / \mathrm{min} / 1.73 \mathrm{~m}^{2}$ $(n=46)$. In general, a smaller proportion of dialysed children achieved the standards set compared with those who had a transplant: haemoglobin, calcium, phosphate and parathyroid hormone target ranges were achieved in $43.9,75.2,49.1$ and $36.3 \%$, respectively, of dialysed children, compared with $47.8,95.7,77.8$ and $70.6 \%$, respectively, of transplanted children. For bicarbonate values, similar proportions of dialysed and transplanted children achieved the set target: $75.2 \%$ compared with $73.9 \%$.

A survival analysis in this year's report included 1,575 children aged $<16$ years between 2003 and 2016 with at least 1 year of follow-up: this showed a total of 84 deaths. As in previous years, younger children had the worst survival, with $88.5 \%$ (95\% CI $84.0-91.9 \%)$ alive at 3 years, compared with $97.5 \%$ in the 12 to $<16$ years age group (95\% CI 94.9-98.8\%; Fig. 3). 


\section{Conclusion and Future Analyses}

This year's paediatric chapter focused on reporting key demographic and biochemical variables with good completeness for children receiving RRT for ESKD in the United Kingdom. We are grateful for the ongoing efforts of paediatric centres to submit data to the UKRR in a timely fashion, which enables thorough data checks and validation prior to publication. The UKRR is keen to expand upon data reported from the core paediatric dataset and is working with the Renal Association Clinical Services Committee to identify priorities for data collection, audit and research. Dependent on data completeness, areas of interest for inclusion in future reports include reporting data for all young people aged 16-18 years, irrespective of paediatric or adult management, as well as data for children with stages 4 and 5 chronic kidney disease (non-RRT).

\section{Acknowledgements}

The UKRR would like to thank patients for the inclusion of their data and renal centres for submitting the data. The UKRR is grateful to NHS Blood and Transplant for sharing data.

\section{Statement of Ethics}

The 21st UKRR Annual Report was produced in accordance with the provisions and regulations of the government of the United Kingdom and Northern Ireland, the National Health Service in England, Northern Ireland, Scotland and Wales, the Information Commissioner's Office and where applicable the devolved governments of Northern Ireland, Scotland and Wales. Due to the number of patients whose data are included in the annual report, the UKRR relies on provisions under health and social care legislation (both British and devolved) that nullify the requirement for common law consent to be collected for audit purposes.

\section{Disclosure Statement}

The authors have no conflicts of interest to declare.

\section{Author Contributions}

All authors made a substantial contribution to the content included in this summary; participated in drafting and critically revising the summary; approved the final version to be published and agreed to be accountable for all aspects of the work.

\section{References}

1 The Renal Association [Internet]. Guidelines and commentaries [cited June 6, 2019 ]. Available from: https://renal.org/guidelines/.
2 UK Renal Registry. UK Renal Registry 21st Annual Report - data to 31/12/2017. Bristol, UK; 2019.
3 Schwartz GJ, Muñoz A, Schneider MF, Mak RH, Kaskel F, Warady BA, et al. New equations to estimate GFR in children with CKD. J Am Soc Nephrol. 2009 Mar;20(3):629-37. 